through the wound, and, being diseased to one-eighth of an inch, a quarter of an inch of it was removed by a Hey's saw. The os calcis was next examined, and the diseased portion, as well as some of the sound bone (to make sure of leaving no disease behind), altogether nearly half an inch of its upper surface, was taken away. Nothing now remaining but healthy structures, the parts were carefully sponged, the edges brought together by a few sutures, water dressing applied, and the linb placed on a double inclined plane. Permanganate of potash was prescribed on account of the hectic symptoms.

The patient improved rapidly. By the middle of January he was able to walk with the help of a stick; and in June, 1868, he came to see me. By wearing a peculiarly constructed boot of his own invention, he was able to walk exceedingly well without any help. The foot presented the appearance shown by the accompanying engraving. The

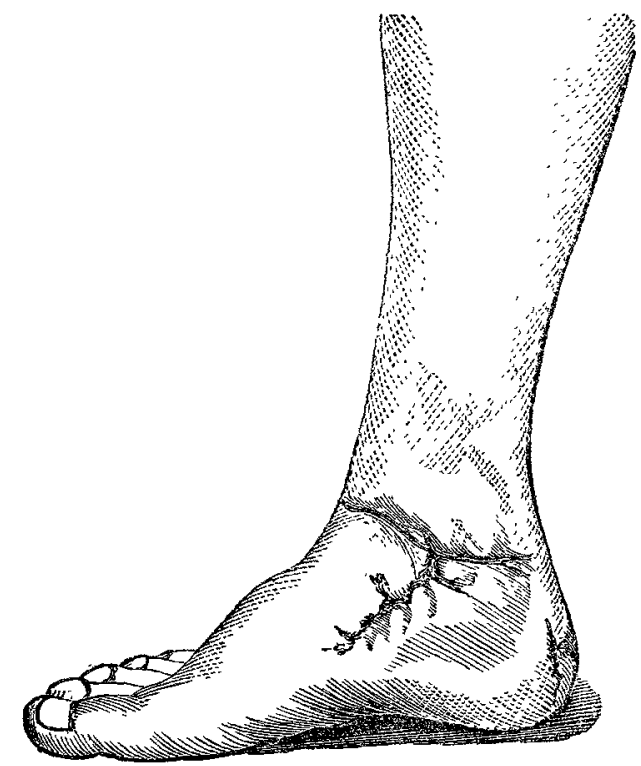

ankle was strong, and, strange to say, was possessed of a considerable and increasing amount of mobility.

Remarks.-This case presents the following points of interest:-

1st. 'The unusually large amount of bone removed.

2nd. The safety with which the joint between the scaphoid and internal cuneiform, and the extensive synovial membrane (which not only lies between them, but also insinuates itself between all the bones of the tarsus, from the cuboid to the second and third metatarsal bones) were opened.

3rd. The disappearance of hectic, and the recovery without a single bad symptom, attributable, in my opinion, to the thorough extirpation of all diseased structures.

4th. Mobility.

The slight injury occasioned to the soft parts by the vertical incision and the two transverse ones was such as emboldens me to recommend this method to the consideration of the profession in those cases where great swelling of the joint exists. In the present case the only part of importance incised was the tendon of the tibialis anticus; the dorsalis pedis and other structures were easily pushed aside and avoided. Extensive gaping will result from the incisions, not sufficient perhaps for an expeditious operation, but enough for cautious and slow manipulation and removal of all diseased bone. If the external malleolus be not, as in this case, previously excised, it might be easily removed by a vertical incision, turning aside the peronei, and applying a bone forceps and knifo.

Sept. 1869.

\section{CASE OF SUPPRESSTON OF URINE CURED BY THE EXTERNAL USE OF DIGITALIS.}

\section{Bx HOWARD D. REYNOLDS, L.R.C.P., M.R.C.S.}

MrS. _- a strong, healthy woman, aged forty-seven, sent for me one evening, and on my arrival she complained of severe pain in the bowels and back, and that she had not voided more than a wineglassful of urine for the last six days. There was no distension of the bladder to be detected. Constant sickness prevailed, which of course I failed to arrest. Pulse 114 per minute; there was slight drowsiness, but no sleep. My patient thought that she had often suffered from gravel, but never required any medicine for it.

I immediately commenced my treatment, such as sweating, warm applications to the parts affected, and the internal use of digitalis, in one-grain doses, every four hours, up to the evening of the eighth day, without any satisfactory result, except that the pulse came down to 80 per minute. I then resolved to try digitalis externally, having heard J. D. Brown, Esq., of Haverfordwest, whose pupil I had been, and who was, I believe, the first who published a case of suppression treated by digitalis externally, mention a case many years ago treated in this way. I at once despatched two persons to collect some digitalis: although late in the evening, they returned with a great quantity. I immediately had the leaves made into a sort of pulp or ponltice, by pouring boiling water over them, and applied the first poultice about 11 P.M., which was kept on four hours, when it was removed and a fresh one supplied. Two hours afterwards a pint of clear, pale urine was passed. A fresh poultice was again applied, and the patient was carefully watched. Plenty of urine continued to be voided, and the patient gradually recovered, and is now quite well.

Pembroke Dock, Oct. 1869 .

\section{THE CASE OF LEOPOLD I., KING OF THE BELGIANS.}

BY THE LATE M. CIVIALE,

UTMBER OF THT INSTITUTE OF FBANCH.

To the Editor of The LANCET.

Srr,-Accident has placed in my hands the case of the late King of the Belgians, drawn up by M. Civiale. The authenticity is undoubted; and as the case cannot fail to interest many of your readers, 1 offer it to you for pablication in The Lancet.

I am, Sir, yours \&c.,

St. James's-place, Nor. 1st, 1869.

WAITER J. Cotzson.

The following case is worthy of attention in many points of view. It is the most difficult and instructive one that I have met with during the course of my long practice, and I feel that I may communicate it to the profession, although it refers to his late Majesty Leopold I., King of the Belgians.

Long before I was called on to attend the izing, his Majesty had suffered under symptoms indicating disorder of the urinary organs, although the exact cause remained unknown. Retention of urine occurred. A catheter was introduced, and a small concretion came away in tie eye of the instrument. Several other concretions (gravel) were subsequently discharged with the urine.

His Majesty then set out for Biarritz, but was compelled to stop on the way. The digestive organs became extremely deranged. The urine contained a quantity of mucus, was stained with blood, and voided with difficulty. These symptoms were relieved after some time, but they returned as soon as the journey was resumed, and were alwajs aggravated by prolonged exercise.

About the end of 1861 his Majesty experienced a very severe attack in London. The surgeons who were consulted on this occasion were of opinion that the symptoms depended on calculus in the bladder, and Sir Benjamin Brodie recommended that I should be called in.

On the 1 st of March, 186: I paid my first visit to his Majesty at Lacken. I then ascertained from the patient himself that he had formerly suffered under stricture of the urethra, for which dilatation and canterisation had been employed. It was evident, however, that some ot 1 er cause was to be. sought for the serious symptoms alrendy alladed 